\title{
The performance improvement of look-alike sound-alike drug pre-dispensing
}

\author{
Manutchanok Jongprasithporn ${ }^{1, *}$, Wichai Sunkarat ${ }^{1}$, Thanakorn Homsai ${ }^{1}$, Teppakorn Sittiwanchai ${ }^{2}$ \\ and Nantakrit Yodpijit ${ }^{2}$ \\ ${ }^{1}$ Department of Industrial Engineering, Faculty of Engineering, King Mongkut's Institute of Technology Ladkrabang, \\ Bangkok, Thailand \\ ${ }^{2}$ Center for Innovation in Human Factors Engineering and Ergonomics (CIHFE^2), Department of Industrial Engineering, \\ Faculty of Engineering, King Mongkut's University of Technology North Bangkok, Bangkok, Thailand
}

\begin{abstract}
Medical dispensing errors are common and classic human errors in the hospitals. Lookalike, Sound-alike (LASA) drug is one of the main issues in pre-dispensing and dispensing processes. The data in this study were observed from one middle size hospital in Thailand. In 2017, there were two months that the medication errors KPI $(0.065$ and 0.061$)$ were greater than the accepted error threshold (0.06). The SBAR (Situation, Background, Assessment, and Recommendation) solution and HOSxP software were applied for three months in the predispensing process to reduce the medical dispensing errors. The results showed that the medication errors $\mathrm{KPI}(0.04,0.03$, and 0.02 , respectively) were reduced dramatically. Therefore, implementation of the SBAR and HOSXP software is recommended as a fast and easy solution for medical dispensing errors.
\end{abstract}

Keywords - LASA; Human errors; Pre-dispensing process

\section{Introduction}

Pre-dispensing error and dispensing error are defined as a misunderstanding of dispensed processing between prescription and pharmacist [1]. The errors of pharmacist are incorrectly dispensing dose as specified in the prescriptions, such as medical type, dosage form, dosage, medical size, administration, number of prescriptions. The errors are also included dispensing without prescriptions such as dilution/ incorrect mixture using improper container, incorrect labeling, having wrong patient name, drug name and so on [2].

Look-alike sound-alike (LASA) drug is medications that could make people confuse because of their appeal or name. LASA can cause a confusing and medication error. In sound-alike, medications have common names or trade names with similar written, spelling, phonetics. In look-alike, medication products have similar color and size of tablets, bottles, labels or packaging to other drugs or similar to the same drug with different strength [3].

In 2007, world health organization (WHO) published "Nine patient safety solutions". LASA is one of the nine topics in the Nine patient safety solutions document [4]. It provided guidance about the prevention of LASA problems to ensure the safety of patients. WHO also distributed a guideline to managing LASA problems in member countries. In Thailand, the ministry of public health announced the national patient safety policy during 2007-2008 [5]. There are two main issues that are focusing on preventing infection from medical treatment and drug safety. In drug safety issue, there are three points that need to concern such as high alert drug, drugs that cause severe adverse reactions and repeatedly allergic drug. The pharmacist polls indicated that $82.46 \%$ of problems in hospitals came from LASA drug. LASA is the first rank causing of the medication errors [6]. In 2009, the drug system situation was reported that the drug safety related to LASA drug. A survey of 25 hospitals found the first rank problem of LASA drug was caused by a medicine labels and drug bottles from the same company. The second rank problem was the drug tablets that were looked alike and had similar brand name to other drugs.

Several studies [7] suggested that solving LASA drug problem in hospitals is continually processing by not buying look-alike and sound-alike drug. Putting the caution sign on the drug packet is another method to remind for the patients. However, medication error problem from LASA drug still cannot avoid because of physical limitations of human such as visual problem which could effect on choosing the wrong type of drug [8]. Therefore, the purpose of this study is to apply human factors engineering and human information processing to solve LASA problem in a pharmacy department in the hospital.

The objective of this paper is focused on the transcribing process of a pharmacist. The transcribing

*Corresponding author: kjmanutc@kmitl.ac.th 
process is a part of the pre-dispensing process and it is one of the risk processes causing the medical errors, directly related to human. In this paper, the SBAR (Situation, Background, Assessment, and Recommendation) solution [9] is used along with HOSxp program. The HOSxP is a software providing to connect the medicine data in the hospital database in order to label the assigned medicine labels.

\section{The Study of Transcribing Error and Objective}

As described earlier, the scope of this study was to focus on a part of the transcribing process of pharmacists. The transcribing process is directly related to the model of human information processing (HIP) [10]. HIP model shows that the working memory is involved in decision-making from sight and hearing of human. Attention also play an important role to perception, working memory, decision making and response execution while one is dealing with look-alike, sound-alike medications.

The FDA U.S journal indicated that "miscommunication of drug orders can involve poor handwriting, confusion between drugs with similar names, poor packaging design, and confusion of metric or other dosing units". The content in the journal was specified that the poor handwriting of a doctor was affected by the transcribing error of pharmacists. Moreover, it can lead to the pre-description errors. The issue about unavoidable of LASA drug from one of the private hospital in Thailand was studied in this study.

Figure 1 shows the example of Look-Alike, SoundAlike medication that can cause transcribing error. Both medications have the same ending name, which is CILLIN. They also have similar bottle and same amount $500 \mathrm{mg}$. Key Performance Indicator (KPI) is one of the techniques, used for a performance appraisal in order to achieve the expected value. In this case, the expected KPI should be less than 0.06 that means the errors should be less than 60 per 1,000 numbers of visits. The data was collected in 2017 from a sampling hospital. The blue line is the occurred errors and the red line is the hospital error threshold (0.06).

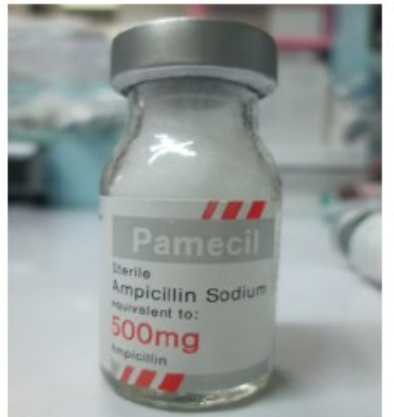

(a)

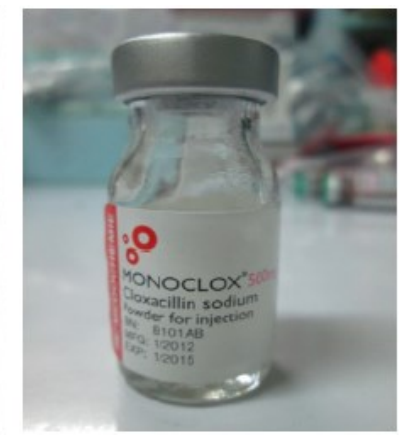

(b)
Fig. 1. Look-Alike, Sound-Alike Medication (a) Ampicillin $500 \mathrm{mg}$ (b) Cloxacillin $500 \mathrm{mg}$ [11]

In 2017, a hospital's KPI (Figure 2) showed that the occurred medication errors in February and July
(0.065 and 0.061 , respectively) were greater than the hospital error threshold. In March and May, there were the occurred medication errors, equalling to hospital error threshold. When KPI value was equal or greater the error threshold, it could indicate that patients might have high risks to get effect by medication errors. Patients who receive wrong dose and/or wrong medicine could be harmful to their life. Hence, the methods to preventing LASA medication errors had been an important issue for the hospitals.

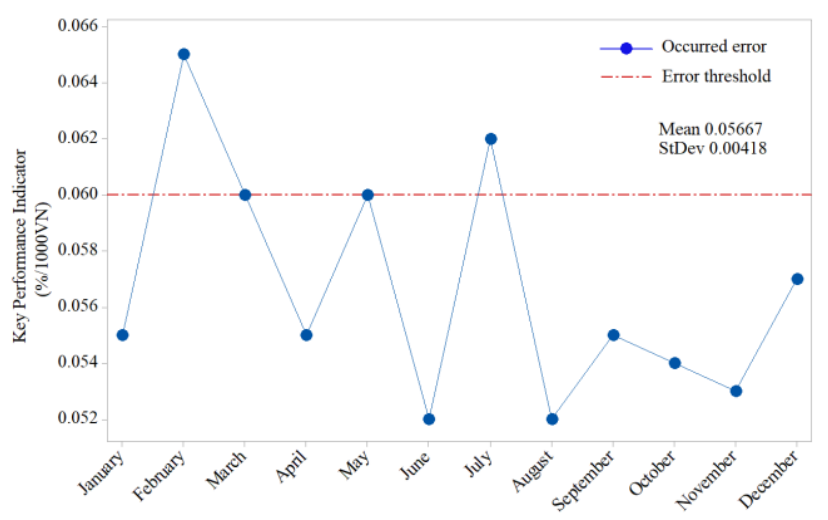

Fig. 2. Occurred transcribing error of a sample hospital in 2017

To improve LASA, this study is focusing on the SBAR (Situation, Background, Assessment, and Recommendation) and HOSxP software. The SBAR is a tool for rechecking the medical prescription and it is used for practicing in taking and giving report among nursing students [12]. In Situation (S), healthcare givers have to describe the specific situation including the patient's name, consultant, patient location, code status, and vital signs. In Background (B), healthcare givers have to inform patient's background including admitting diagnosis, date of admission, prior procedures, current medications, allergies, pertinent laboratory results and other relevant diagnostic results. In Assessment (A), healthcare givers have to think critically about the reason for your patient's condition including laboratory results. In Recommendation ( $R$ ), healthcare givers have to inform about what you would like to happen by the end of the conversation with the physician and clarify your expectations.

The HOSpital eXPerience (HOSxP) is a free software, developed by Thai programmer [13]. It has been used by 150 health centers and mid-size hospitals in Thailand. The advantage of the HOSxP program is that the front of the medical label can be adjusted such as resize, making bold letter and changing lower case to upper case and so on. The writing of drug's name in upper case letter can help distinguish LASA drug.

\section{Research Methodologies}

There are two main methodology parts in this study, including observing of a pre-dispensing procedure and improving process.

The pre-dispensing procedure was summarized as shown in Figure 3. The pre-dispensing procedure was 
including five steps. First, the prescription was written by doctor's handwriting. Second, the lists of the medicine were transcribed by a pharmacist. Third, the lists of medicine were prepared in the pre-dispensing process. Fourth, the medicines of the patients were checked in the validity checking process. In the fourth step, if the pharmacist found incorrect prescription, the lists of medicine will be sent to transcribing process again. The lists of the medicine were sent to the dispensing process when the prescription was correct. The depended medicine was recorded and reported to the doctors.

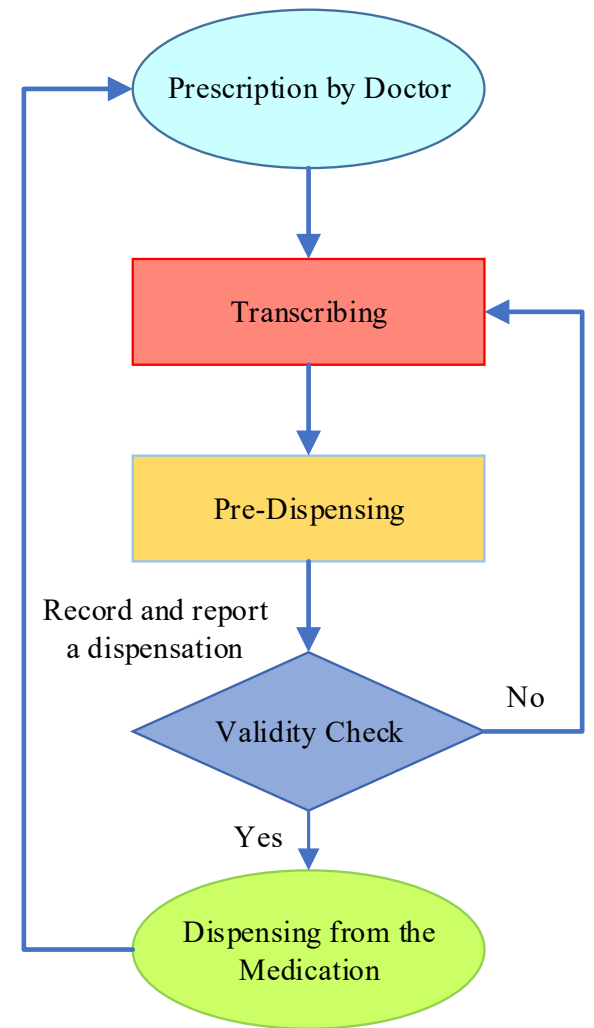

Fig. 3. Flow-chart of pre-dispensing procedure without the proposed solution

The problem can be divided into three main issues. The first problem was identified as a communication issue. Miscommunication between doctors and pharmacists was one of the most important LASA issues. The problem occurred when pharmacists could not recognize handwriting of doctor. The second issue was lacking of the pre-dispensing process skill of new pharmacists. This issue could happen when the pharmacy department admitted new pharmacists who had less experience. The third issue was related to a software. Normally, the efficient pharmacy software for medicine management is very costly to hospitals. Therefore, reducing medication errors were limited by the software cost. For example, using of a normally the labelled software the LASA medicine label cannot be labelled in a Tallman letter. The Tallman letter is the using of upper/lower case letter or different letter size to prevent the medical error.

A flow chart of pre-dispensing procedure with SBAR and HOSxP was described in Figure 4. To solve the transcribing errors, the SBAR (Situation,
Background, Assessment, and Recommendation) and HOSxP software were applied in the pre-dispensing procedure for three months from January to March, 2018. The SBAR and HOSxP software were added between the prescription by doctors and transcription by pharmacists. The prescriptions have been written by the doctor's handwriting. The SBAR could help enhance communication between doctors and pharmacists and ensure the correct lists of medication before it was sending to the pre-dispensing process. In the SBAR process, an unreadable prescription was checked with the previous hospital database of the patients. The prescription was rechecked by a pharmacist in transcribing process. Then, the checked prescription was sent to the doctor again for authentication. In the HOSxP process, the authorized prescription was labeled by HOSxP software and sent out to pre-dispensing process. The redundant visually check was implemented in the improved process to increase patient's safety. Although SBAR and HOSxP were added to reduce human errors, the lists of medicine still needed to check in the validity checking process again. Then the lists of the medicine were sent to the dispensing process.

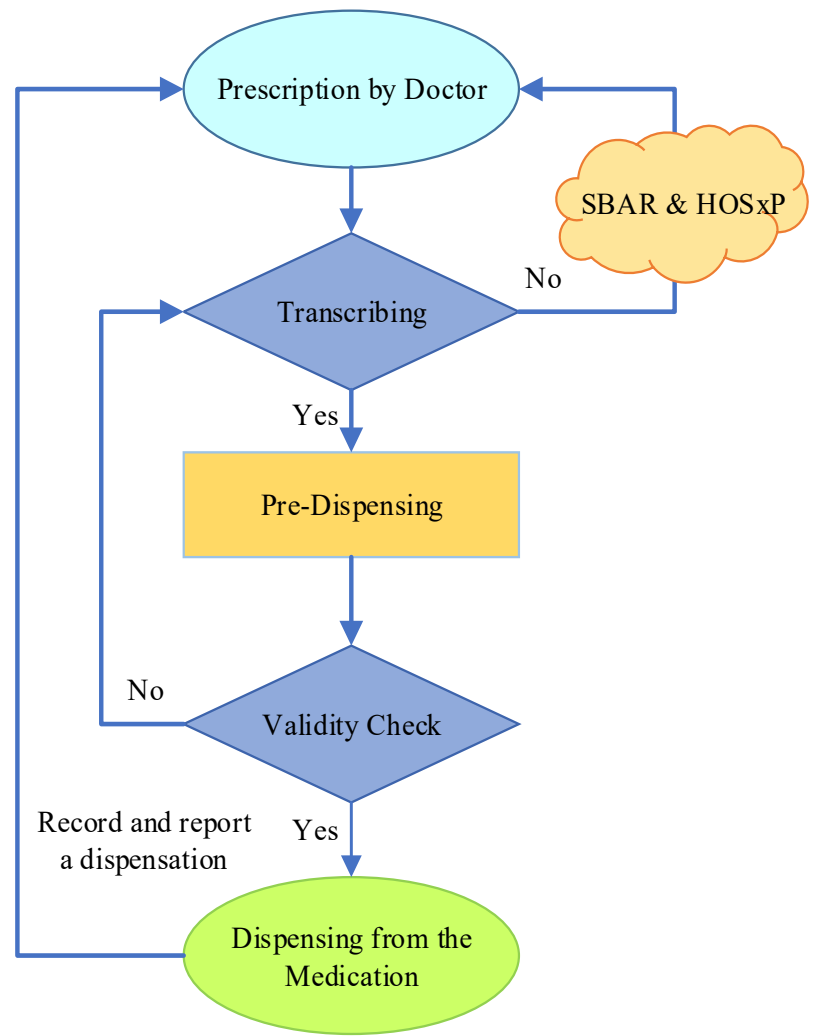

Fig. 4. Flow-chart of pre-dispensing procedure with the SBAR and HOSx

\section{Results}

After implementing SBAR and HOSxP, the KPI of the transcribing error was presented from January to March, 2018 (0.04, 0.03, and 0.02, respective). The data was compared between before and after applying SBAR solution with HOSxP software between the prescription by doctors and transcription by pharmacists. The average KPI of the transcribing errors were compared as shown 
in Figure 5. It is obvious that the average occurred error in 2018 is less than that of the occurred error in 2017. The expected KPI is that the 0.06. However, before applying the proposed techniques in February and July, the KPI are higher than 0.06. After using the proposed techniques to improve the transcribing process, the KPI is less than 0.04 that means the error from transcribing process is less than 40 visit numbers per 1,000 visit number. It indicated that the proposed solution can decrease the transcribing error efficiently.

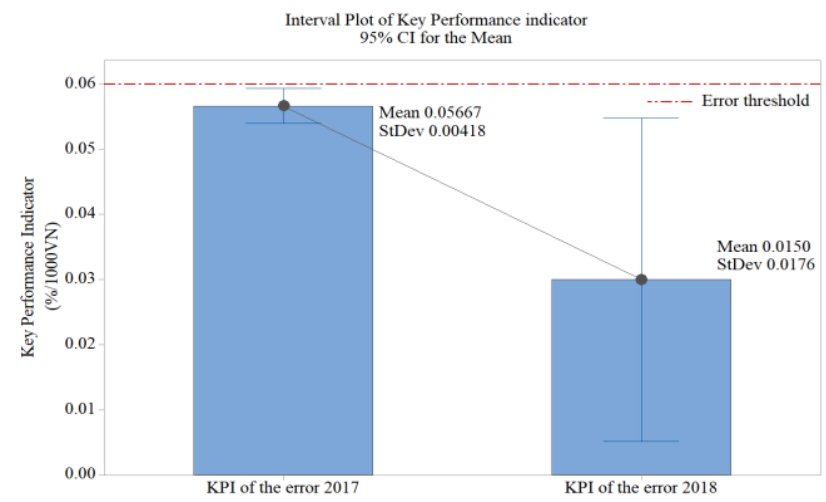

Fig. 5. The compared avearge KPI in 2017 and 2018

\section{Discussions \& Conclusions}

To decrease the medical error from LASA drug, the SBAR solution and HOSxP software are used in the pre-describing process. The results showed that the using of SBAR solution and HOSxP software could improve the pre-dispensing process and decreased the medical errors from LASA drug. Hence, the proposed solution can solve the problems of miscommunication, human errors, and inexperienced pharmacist issues. The HOSxP, an efficient free software is recommended to use in pre-describing process to solve the LASA problem. The advantage of HOSxP software is that the letter in the medicine label can be formatted in Tallman letters. The Tallman lettering can reduce a confusable drug name of LASA drug because using of Tall man lettering in a part of medicine name in upper case letters can help to distinguish LASA drugs.

The limitations of this study are including three issues. The first limitation is the difficulty of connecting between the HOSxP program and the hospital database. Although the HOSxP software is a free software, it requires a specific program to help connect between the HOSxP and the hospital database. The second limitation is lacking pharmacists' skill and experience in predispensing process. The pharmacists' skill and experience is one of the most important factors in order to solve LASA problem. The pharmacists who is lacking of skill and working experience could cause the transcribing error. The last limitation is the issue about the hospital confidential information. Therefore, it is difficult to provide some results in the public.

In the future, the doctor should type prescriptions instant of writing in the prescription process. Moreover, the hospitals should have their own prescription program, which enhances data communication between doctors and pharmacists.

\section{References}

1. W. T. Basco; M. Ebeling; T.C. Hulsey; K. Simpson, "Using Pharmacy Data to Screen for Look-Alike, Sound-Alike Substitution Errors in Pediatric Prescriptions", Academic pediatrics, vol 10, issue 4, pp. $233-237,2010$

2. T. Nagata, M. Kimura, F. Tsuchiya, "Similarity Index for Sound-Alikeness of Drug Names with Pitch Accents", Procedia Computer Science, vol 35, pp 1519-1528, 2014

3. N. Tuohy, S. Paparella, "Look-Alike and SoundAlike Drugs: Errors Just Waiting to Happen", Journal of Emergency Nursing, vol 31, Issue 6, pp. $569-571,2005$

4. World Health Organization. WHO launches 'Nine patient safety solutions' [online]. 2007 [cited Oct 7,2016]. Available from: www.who.int/mediacentre/news/releases/2007/pr22/ en/ index.html.

5. Department of Health Service Support. National patient safety goal. Nonthaburi: Ministry of Public Health: 2007-2008.

6. Chumchit C. Development of medication safety management system for Look-Alike Sound-Alike drugs in public hospital [dissertation]. Nakorn Pratom: Silpakorn University; 2013.

7. Nursing Students' perspectives of the Cause of Medication errors. Nurse Education Today 34 (2014) 434 - 440, April 16,2013.

8. Academic Centers for Drug System Surveillance and Development. Drug system situation report [online]. 2009 [cited Oct 7,2016]. Available from: www.thaidrugwatch.org/download/ Other/print/otherprint01.pdf

9. Thomas CM, Bertram E, Johnson D. The SBAR communication technique: teaching nursing students professional communication skills. Nurse Educ. 2009;34(4):176-80. Www.ncbi.nlm.nih.gov/pub $\mathrm{med} / 19574858$

10. Wickens,C.D. (1984). Processing resources in attention. In R. Parasuraman \& D.R. Davies(Eds.), Varieties of attention(pp.63-102). New York: Academic Press

11. U.S. Food and Drug Administration, [online] Available in https://www.fda.gov/ForConsumers/ConsumerUpda tes /ucm048644.htm

12. R. Charuwanno, J. Vongchanglaw, $T$. Pongsananurak, "Effects of Teaching by Using SBAR Technique on Knowledge Attitude and Practice in Taking and Giving Report Among Nursing Students" Vol 15, No 3, pp 690-397, 2014

13. HOSxP, [online] Available: http://hosxp.net/. 